\title{
Use of Marine biotoxins to modulate the tyrosine kinase domain of the human epidermal growth factor receptor
}

\author{
Charli Deepak Arulanandam ${ }^{1}$, Ramesh Dharmaraj ${ }^{2}$, Prathiviraj Ragothaman ${ }^{3}$, Prakash \\ Vincent Samuel Gnana ${ }^{4 *}$ \\ ${ }^{1}$ Department of Medicinal and Applied Chemistry, Kaohsiung Medical University, Kaohsiung - 80708, Taiwan \\ ${ }^{2}$ Department of Animal Health Management, Alagappa University, Karaikudi - 630003, India \\ ${ }^{3}$ D Department of Microbiology, Pondicherry University, Puducherry, India. \\ ${ }^{4}$ Centre for Marine Science and Technology (CMST), Manonmaniam Sundaranar University, Rajakkamangalam, Kanyakumari, 629502, India \\ *Correspondence should be addressed to Prakash Vincent Samuel Gnana; e-mail: prakash@msuniv.ac.in
}

\section{Abstract}

Inappropriate activation of the Epidermal growth factor receptor (EGFR) group of kinases has been identified in a variety of tumour cells, either due to mutation or overexpression. Although the tumour is a fatal disease, significant therapy discoveries have lately been made. The human EGFR and this family of kinases have emerged as promising targets for cancer therapy. In this molecular docking study, Natural marine toxins are employed to regulate the activity of the human EGFR tyrosine kinase domain (EGFRtkd) in the molecular docking investigation (PDB ID5JEB). Marine biotoxins can cause neurological, gastrointestinal, and cardiovascular problems, as well as severe mortality and long-term morbidity in some situations. Because there is no antidote for any of the natural marine poisons, supportive care is the mainstay of treatment. Paralytic shellfish poisoning, in particular, and puffer fish poisoning, in particular, can result in death within hours of exposure to the poisons and may require immediate medical intervention. However, this research found that marine biotoxins can modulate EGFRtkd. Furthermore, homoyessotoxin was anticipated to be an EGFRtkd modulator with a binding affinity as $-9.584 \mathrm{kcal} / \mathrm{mol}$. To employ the homoyessotoxin in tumour therapies, further knowledge of natural marine biotoxins and further toxicological research is required. 
Introduction

Epidermal Growth Factor Receptor (EGFR) is a transmembrane glycoprotein receptor for epidermal growth factor. When EGFR interacts to its cognate ligand EGF, it triggers tyrosine phosphorylation and receptor dimerization with other family members, resulting in uncontrolled cell proliferation. Several anti-EGFR medicines such as monoclonal antibodies and tyrosine kinase inhibitors, have been developed, allowing doctors to identify and treat specific patient groups. (Seshacharyulu et al., 2012). EGFR is a protein located on the surface of some normal cells that help them develop. It's also possible that its present in high concentrations on cancer cells, causing them to multiply and divide (www.cancer.gov). EGFR and EGF-like peptides are widely overexpressed in human carcinomas, and in vivo and in vitro studies have shown that these proteins can accelerate cell transformation (Mendelsohn and Baselga 2000). The EGFR signal is part of a complex network that has been successfully treated as a tumour target (Oda et al., 2005). The known EGFR inhibitors show varying antitumor responses for the various EGFR mutation connected to nonsmall-cell lung cancer (Bethune et al.,2010; Sogabe et al., 2012). If the EGFR is suppressed tumour cells may not be able to multiply. In other cases, EGFR inhibitors were used to treat the malignancy (www.cancer.gov). Natural marine toxins have been tested against the EGFRtkd in DockThor-VS web portal as part of current reseach (Guedes et al., 2021). The goal of this study is to use computational tools to assess natural marine biotoxins in order to alter the EGFRtkd function. Toxic compounds exist in a variety of shapes and sizes, and there are a variety of methods for identifying them, including biological origins, toxicity, molecular mass, and structural analysis. Marine biotoxins are produced by toxicogenic algae, cyanobacteria, bacteria, and marine animals. (Bigalke and Rummel, 2005; Johnson et al., 2011).Natural marine toxins (non-protein) in man and animals induce amnesic shellfish poisoning (ASP), azaspiracid shellfish poisoning (ASP), diarrheic

54 shellfish poisoning (DSP), neurotoxic shellfish poisoning (NSP) and paralytic shellfish 
poisoning (PSP) (Lowenstine 2008). The first toxic component of paralytic shellfish poisoning to be found and studied (PSP) was saxitoxin (STX) (Wang et al 2015).This toxin inhibits neuronal transmission by binding to the voltage -gated Na+ channel (Andrinolo et al., 1999). According to research published in the late 1980s, inhalation delivery of STX to mice is around 10 times more effective than intravenous treatment (Neufeld 1980). STX is similar to lowmolecular-weight toxins like tetrodotoxin, brevetoxin, and anatoxin in term of its ability to permeate the dermal barrier (Kubo et al 2008). The human percutaneous lethal dose of saxitoxin is unknown, although the equivalent brevetoxin worksin a similar way. Experiments have revealed that a percutaneous dosage of brevetoxin 20 times larger than the deadly parenteral dose does not cause death. (Supotnitskiy, 2013).

\section{Methodology}

\section{Targeted Enzyme}

EGFRtkd (PDB ID-5JEB) was chosen for this molecular docking investigation. It can be found at www.rcsb.org in the RCSB protein database. This EGFRtkd has been linked to a variety of cancers and plays a crucial role in cellular processes (Sogabe et al., 2012).

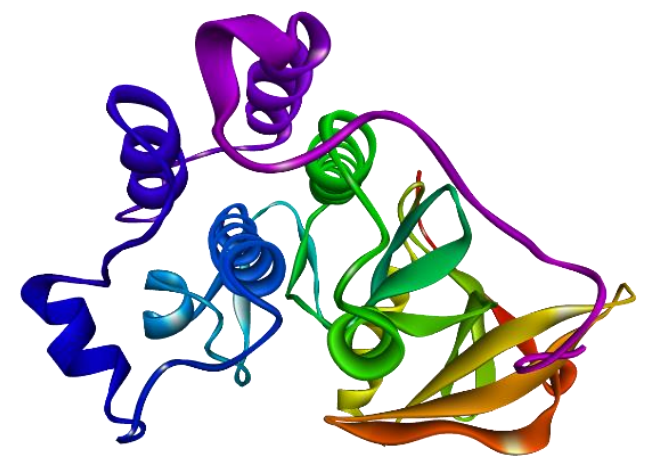


75 The DockThor-VS web server, as well as curated libraries of currently accessible drugs and chemical data, are publicly available at www.dockthor.lncc.br. Its molecular docking and

77 virtual screening experiments are simplified. This website was used to evaluate the natural marine toxins in order to find an EGFRtkd modulator.

\section{Results and Discussion}

80 Homoyessotoxin binds to the targeted receptor EGFRtkd with exceptional affinity. Fifty 81 marine biotoxins 3D files based on KEGG chemical information were collected from the

82 ChemSpider database. Eight compounds (\#) were not processed in the chosen docking method due to an unknown fault (Table 1). Homoyessotoxin had the lowest binding affinity of all the ligands examined, at $-9.584 \mathrm{kcal} / \mathrm{mol}$. The BIOVIA Discovery Studio Visualizer software was used to visualize the interactive $2 \mathrm{D}$ posture of the receptor with homoyessotoxin docked file. Figure 2 shows the receptor-ligand interaction pattern.

87

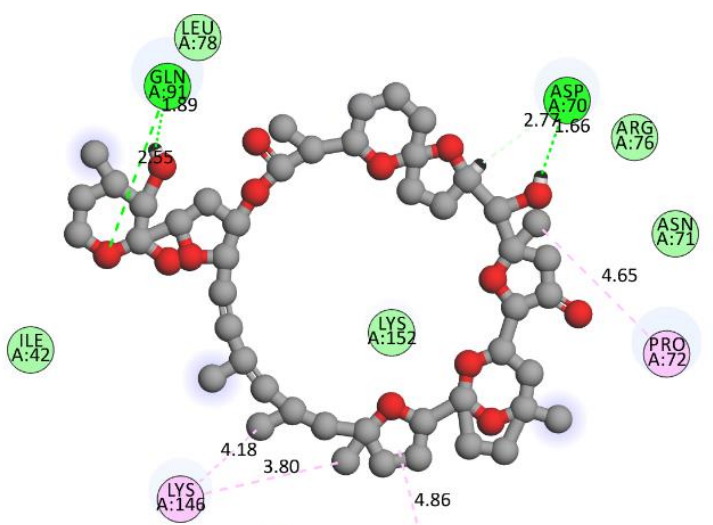

(T:14R)

Figure 2. Receptor-Ligand (EGFRtkd-homoyessotoxin) interaction pattern in 2D

As a contaminant, homoyessotoxin is found in the digestive glands of Mytilus galloprovincialis (Satake et al 1997). It is potentially biotoxin (https://hmdb.ca/metabolites/HMDB0036615) and belongs to the class of organic, lipid-soluble polyether compounds consisting of 13 to 14 rings 
92 fused by ether links into a highly stiff ladder-like structure. According to a 2003 study by

93 Tubaro et al., homoyessotoxin treated female mice died at a dosage of $375 \mathrm{mg} / \mathrm{kg}$. The mice

94 were restless and leapt before death, but necroscopy revealed no substantial alterations. The

$95 \mathrm{LD}_{50}$ value of homoyessotoxin-treated mice is $315-830 \mathrm{mg} / \mathrm{kg}$. At a dose of $1 \mathrm{mg} / \mathrm{kg}$, Oral

96 administration of homoyessotoxin to mice did not cause any significant difference in plasmatic

97 enzymes (AST, ALT, LDH and CK) or leukocyte percentage. In mice treated with

98 homoyessotoxin at a dose of $1 \mathrm{mg} / \mathrm{kg}$, cytoplasmic protrusions of myocardiocytes, packed

99 rounded mitochondria, and fibre alterations were seen using transmission electron microscopy

100 (TEM) (Tubaro et al 2003).

\section{Funding}

102 This research received no funding from public, private, or non-profit organization.

\section{Acknowledgments}

104 The author would like to thank for the International Student fellowship from Kaohsiung

105 Medical University (KMU). The author extends his appreciation to Office of Library and

106 Information Services (OLIS) for providing the access to the monographs, journals, internet

107 sources, digital materials as well as other useful software.

\section{Conflicts of Interest}

109 The author declared as there are no known competing financial interests.

\section{References}

111 1. Bethune, G., Bethune, D., Ridgway, N. \& Xu, Z. Epidermal growth factor receptor (EGFR) in lung 112 cancer: an overview and update. J. Thorac. Dis. 2, 48 (2010).

113 2. Bigalke, H. \& Rummel, A. Medical aspects of toxin weapons. Toxicology 214, 210-220 (2005).

114 3. Guedes, I. A. et al. Drug design and repurposing with DockThor-VS web server focusing on SARS- 
4. Kubo, T., Kato, N., Hosoya, K. \& Kaya, K. Effective determination method for a cyanobacterial neurotoxin, $\beta$-N-methylamino-l-alanine. Toxicon 51, 1264-1268 (2008).

5. Lowenstine, L. J. Algal bloom toxicity in marine animals. in Zoo and Wild Animal Medicine 341-348 (Elsevier Inc., 2008).

6. Mendelsohn, J. \& Baselga, J. The EGF receptor family as targets for cancer therapy. Oncogene 19, $6550-6565$ (2000).

7. Neufeld, E. Insects as Warfare Agents in the Ancient Near East (Ex. 23: 28; Deut. 7: 20; Josh. 24: 12; Isa. 7: 18-20). Orientalia 49, 30-57 (1980).

8. Normanno, N. et al. Epidermal growth factor receptor (EGFR) signaling in cancer. Gene 366, 2-16 (2006).

9. Oda, K., Matsuoka, Y., Funahashi, A. \& Kitano, H. A comprehensive pathway map of epidermal growth factor receptor signaling. Mol. Syst. Biol. 1, 10-2005 (2005).

10. Satake, M., Tubaro, A., Lee, J. \& Yasumoto, T. Two new analogs of yessotoxin, homoyessotoxin and 45-hydroxyhomoyessotoxin, isolated from mussels of the Adriatic Sea. Nat. Toxins 5, 107-110 (1997).

11. Seshacharyulu, P. et al. Targeting the EGFR signaling pathway in cancer therapy. Expert Opin. Ther. Targets 16, 15-31 (2012).

12. Sogabe, S. et al. Structure-based approach for the discovery of pyrrolo [3, 2-d] pyrimidine-based EGFR T790M/L858R mutant inhibitors. ACS Med. Chem. Lett. 4, 201-205 (2013).

13. Tubaro, A. et al. Oral and intraperitoneal acute toxicity studies of yessotoxin and homoyessotoxins in mice. Toxicon 41, 783-792 (2003).

15. Wang, Q. et al. A novel and functional assay for pharmacological effects of marine toxins, saxitoxin and tetrodotoxin by cardiomyocyte-based impedance biosensor. Sensors Actuators B Chem. 209, 828- 
144 Table 1. Marine biotoxins binding affinity towards the EGFRtkd

\begin{tabular}{|c|c|c|c|c|c|c|c|}
\hline Marine biotoxins & $\begin{array}{c}\text { KEGG } \\
\text { compound } \\
\text { entry }\end{array}$ & $\begin{array}{c}\text { ChemSpider } \\
\text { ID }\end{array}$ & $\begin{array}{l}\text { DockThor } \\
\text { Ligand ID }\end{array}$ & $\begin{array}{l}\text { Affinity } \\
\text { kcal/mol }\end{array}$ & $\begin{array}{c}\text { Total } \\
\text { Energy } \\
\text { kcal/mol }\end{array}$ & $\begin{array}{c}v d W \\
\text { Energy }\end{array}$ & $\begin{array}{c}\text { Elec. } \\
\text { Energy }\end{array}$ \\
\hline \multicolumn{8}{|c|}{ ASP-Amnesic Shellfish Poisoning } \\
\hline Domoic acid ${ }^{l}$ & C13732 & 4445428 & $29 \mathrm{f} 824 \mathrm{cb} 20$ & -6.523 & -18.264 & -1.934 & -39.493 \\
\hline 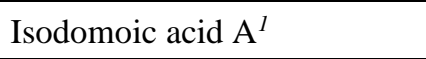 & C20027 & 4946671 & dc8fd6f151 & -6.511 & -21.231 & -0.094 & -37.479 \\
\hline \multicolumn{8}{|c|}{ CFP-Ciguatera fish poisoning } \\
\hline Ciguatoxin $\mathrm{I}^{2}$ & C16762 & 4470834 & $60 \mathrm{c} 8 \mathrm{~d} 84797$ & -9.353 & 107.467 & -29.313 & -8.358 \\
\hline Caribbean ciguatoxin $1^{2}$ & $\mathrm{C} 20003$ & 10216856 & b7c1db87ea & -7.402 & 244.765 & -14.617 & -41.382 \\
\hline CTX 3C ${ }^{2}$ & C20001 & 10474447 & $\mathrm{f} 40814364 \mathrm{c}$ & -8.011 & 155.555 & -18.881 & -25.986 \\
\hline Ciguatoxin $4 \mathrm{~A}^{2}$ & $\mathrm{C} 20002$ & 35013551 & $05 \mathrm{a} 6301 \mathrm{f} 4$ & -8.788 & 149.372 & -25.105 & -16.692 \\
\hline Gambieric acid $\mathrm{A}^{2}$ & C16885 & 27025948 & b9e44b0f68 & -8.910 & 184.602 & -20.454 & -22.981 \\
\hline Gambieric acid $B^{2}$ & C16886 & 9978891 & $5 \mathrm{~d} 70125 \mathrm{~b} 18$ & -9.404 & 194.311 & -25.747 & -10.070 \\
\hline Gambieric acid $\mathrm{C}^{2}$ & C16887 & 10478379 & 0f4d9a62ae & -6.648 & 84.965 & 5.974 & -91.040 \\
\hline Gambieric acid $\mathrm{D}^{2}$ & $\mathrm{C} 16888$ & 10478380 & 6a798aec75 & -7.261 & 102.434 & 3.392 & -80.865 \\
\hline Gambierol $^{2}$ & $\mathrm{C} 20004$ & 4946332 & e7d11eb189 & -8.581 & 69.041 & -24.837 & -11.382 \\
\hline Gambiertoxin $4 \mathrm{~B}^{2}$ & C16852 & 10366292 & 2893bae71e & -8.220 & 164.485 & -7.030 & -47.625 \\
\hline Maitotoxin $^{2}$ & C16854 & 25991548 & $\#$ & $\#$ & $\#$ & $\#$ & $\#$ \\
\hline \multicolumn{8}{|c|}{ DSP-Diarrhetic shellfish poisoning } \\
\hline Okadaic acid ${ }^{3}$ & $\mathrm{C} 01945$ & 393845 & 637352dadc & -8.263 & 41.959 & -19.833 & -25.331 \\
\hline Acanthifolicin $^{3}$ & $\mathrm{C} 20006$ & 27022422 & $\#$ & $\#$ & $\#$ & $\#$ & $\#$ \\
\hline Dinophysistoxin- $1^{3}$ & C16870 & 16737821 & 7f6357dd05 & -7.367 & 155.527 & 1.837 & -78.085 \\
\hline Dinophysistoxin- $4^{3}$ & $\mathrm{C} 20005$ & 4945455 & $197204824 a$ & -9.115 & 4.377 & -26.492 & -23.601 \\
\hline Homoyessotoxin $^{4}$ & C20011 & 4944614 & 67b9b5ae & -9.584 & 59.942 & -27.055 & -16.251 \\
\hline Yessotoxin ${ }^{4}$ & C16872 & 4945067 & $28 \mathrm{c} 096 \mathrm{~d} 0 \mathrm{f} 8$ & -9.135 & 61.155 & -24.247 & -20.509 \\
\hline Caribenolide $\mathrm{I}^{5}$ & C20007 & 10475898 & b634f6bdf5 & -7.392 & 114.925 & 11.641 & -97.630 \\
\hline Goniodomin $\mathrm{A}^{5}$ & C16899 & 27025933 & c0caa4061c & -6.730 & 119.526 & 18.546 & -75.983 \\
\hline Hoffmanniolide $^{5}$ & C20008 & 29212837 & $44852 \mathrm{~d} 982 \mathrm{e}$ & -7.817 & 24.473 & -17.386 & -29.255 \\
\hline Iriomoteolide- $1 \mathrm{a}^{5}$ & C20009 & 17627054 & c7d5ea4232 & -7.249 & 41.559 & -12.622 & -22.561 \\
\hline \multicolumn{8}{|c|}{ NSP-Neurotoxic Shellfish poisoning } \\
\hline Brevetoxin $\mathrm{A}^{4}$ & C16839 & 9041092 & $378 \mathrm{a} 6 \mathrm{ab} 339$ & -7.923 & 159.938 & -16.731 & -15.583 \\
\hline Brevetoxin $\mathrm{B}^{4}$ & C16857 & 9041149 & $\mathrm{c} 4925 \mathrm{~b} 4 \mathrm{~b} 92$ & -8.812 & 93.453 & -23.852 & -9.887 \\
\hline Brevetoxin $\mathrm{B} 1^{4}$ & $\mathrm{C} 20013$ & 27026682 & $\#$ & $\#$ & $\#$ & $\#$ & $\#$ \\
\hline Brevetoxin $\mathrm{B}^{4}$ & $\mathrm{C} 20014$ & 8805247 & $6 \mathrm{a} 8 \mathrm{f} 3546 \mathrm{ec}$ & -8.838 & 142.169 & -19.682 & -25.245 \\
\hline Brevetoxin $\mathrm{C}^{4}$ & $\mathrm{C} 20015$ & 16736106 & $1404334 \mathrm{e} 93$ & -7.783 & 115.521 & -22.669 & -20.070 \\
\hline Hemibrevetoxin $\mathrm{B}^{4}$ & $\mathrm{C} 20016$ & 4946882 & cefb510d0f & -8.158 & 26.868 & -20.307 & -7.230 \\
\hline \multicolumn{8}{|c|}{ PSP-Paralytic shellfish poisoning } \\
\hline Gonyautoxin- $1^{6}$ & $\mathrm{C} 16855$ & 30791735 & $\mathrm{~b} 70612 \mathrm{~d} 0 \mathrm{a}$ & -6.877 & -83.142 & 0.553 & -49.812 \\
\hline Gonyautoxin- $2^{6}$ & C16856 & 9767779 & 69ff2d0957 & -6.614 & -121.898 & -11.750 & -30.799 \\
\hline Neosaxitoxin $^{6}$ & $\mathrm{C} 17208$ & 19975931 & $\#$ & $\#$ & $\#$ & $\#$ & $\#$ \\
\hline Saxitoxin $^{6}$ & C13757 & 34106 & 4e060a9472 & -6.520 & -96.572 & -8.972 & -25.049 \\
\hline Decarbamoylsaxitoxin $^{7}$ & $\mathrm{C} 20021$ & 19975972 & $\#$ & $\#$ & $\#$ & $\#$ & $\#$ \\
\hline Decarbamoylgonyautoxin- $1^{7}$ & $\mathrm{C} 20022$ & 30790887 & b44366465c & -6.251 & -55.542 & -4.309 & -38.583 \\
\hline Gonyautoxin- $5^{8}$ & C20018 & 94743 & $8 \mathrm{f} 46648 \mathrm{~d} 7 \mathrm{~d}$ & -6.708 & -148.962 & -13.217 & -30.449 \\
\hline Gonyautoxin- $6^{8}$ & $\mathrm{C} 20019$ & 30790889 & ccfa77e515 & -6.842 & -102.071 & -16.309 & -29.148 \\
\hline
\end{tabular}




\begin{tabular}{|c|c|c|c|c|c|c|c|}
\hline Gonyautoxin- $8^{8}$ & C20020 & 94734 & 0566d8faf5 & -7.049 & -176.909 & -15.679 & -31.061 \\
\hline Palytoxin ${ }^{9}$ & C16851 & 9280425 & $\#$ & $\#$ & $\#$ & $\#$ & \# \\
\hline Ostreocin $\mathrm{D}^{9}$ & C20028 & 78445048 & $\#$ & $\#$ & \# & \# & $\#$ \\
\hline Tetrodotoxin $^{10}$ & C11692 & 23106940 & ffc85130ea & -6.453 & -55.305 & -3.087 & -36.841 \\
\hline 11-Deoxytetrodotoxin ${ }^{10}$ & $\mathrm{C} 20026$ & 19989712 & $\#$ & $\#$ & $\#$ & $\#$ & $\#$ \\
\hline \multicolumn{8}{|c|}{ Others } \\
\hline Pectenotoxin-1 & C16871 & 4942381 & de9246fafa & -7.940 & 57.958 & -13.852 & -16.524 \\
\hline Pectenotoxin-2 & $\mathrm{C} 20012$ & 4941948 & $4 d 2 b 95$ & -7.864 & 53.110 & -12.615 & -16.849 \\
\hline Azaspiracid & C16907 & 9724424 & $0873 \mathrm{e} 863 \mathrm{~b} 3$ & -8.706 & 11.182 & -20.035 & -22.681 \\
\hline Gymnodimine & C20025 & 64854205 & $\mathrm{db} 2 \mathrm{~b} 7 \mathrm{~cd} 013$ & -7.064 & 89.227 & -14.932 & -2.517 \\
\hline Neosurugatoxin & C16761 & 29214049 & a6116671 & -7.462 & 41.290 & -13.268 & -30.437 \\
\hline Prorocentrolide & C20023 & 10204061 & $83 f 990486 d$ & -7.959 & 261.979 & -3.700 & -66.466 \\
\hline Prorocentrolide B & C20024 & 10200328 & cbc87dc562 & -8.002 & 333.571 & -1.433 & -74.877 \\
\hline Pyropheophorbide a & C18064 & 10381282 & fee4b0a482 & -7.106 & -2.517 & 36.027 & -91.816 \\
\hline \multicolumn{8}{|c|}{$\begin{array}{l}\text { Note: } 1=\text { Kainoids; } 2=\text { Cyclic polyethers; } 3=\text { Okadaic acid and derivatives; } 4=\text { Cyclic polyethers; } 5=\text { Macrolides; } \\
6=\text { Carbamate derivatives; } 7=\text { Decarbamoyl derivatives; } 8=N-\text { Sulfo-carbamoyl derivatives; } 9=\text { Palytoxins; } 10= \\
\text { Tetrodotoxins; \#=unknown error in ligand preparation and docking. }\end{array}$} \\
\hline
\end{tabular}

145 